\title{
Intradural Lumbar Disc Herniation: A Case Report and Literature Review
}

This article was published in the following Dove Press journal:

Clinical Interventions in Aging

\author{
Chao-Yuan Ge \\ Ding-Jun $\mathrm{Hao}$ (D) \\ Liang Yan \\ Le-Qun Shan \\ Qin-Peng Zhao \\ Bao-Rong $\mathrm{He}$ \\ Hao Hui
}

Department of Spine Surgery, Honghui Hospital, Xi'an Jiaotong University, Xi'an, Shaanxi Province, People's Republic of China
Correspondence: Ding-Jun Hao Department of Spine Surgery, Honghui Hospital, Xi'an Jiaotong University, No. 555 Friendship East Road, Beilin District, Xi'an 7I0054, Shaanxi Province, People's Republic of China

Tel/Fax +86-29-87894724

Email haodingjunspine@126.com
Background: Lumbar disc herniation into the dural space is a very rare phenomenon of degenerative lumbar lesions in the elderly population, and its potential pathogenesis and natural course remain unclear.

Case description: We describe a rare case of intradural lumbar disc herniation. A 68-yearold man presented with progressive lower back pain and radiating pain and numbness in both legs for 3 years. Magnetic resonance imaging revealed a large herniated disc at L4-L5. Posterior discectomy and fusion of the L4-L5 was performed after conservative treatment failed. Intraoperatively, only minimal disc fragments in the epidural space were found after meticulous probing following laminectomy of the L4-L5 vertebrae. The dorsal dura mater was saturated, tense, and bulged at the L4-L5 levels; additionally, an intradural mass was palpable and confirmed by intraoperative ultrasonography. Subsequently, dorsal middle durotomy was performed. Upon opening the dural sac, a large cauliflower-like mass similar to nucleus pulposus tissue was found near the arachnoid membrane. The mass was dissociative and could be completely resected. The dorsal dural incisions were closed after careful exploration, followed by fixation and fusion of the L4-L5 levels. Pathological examination revealed disc tissue with central balloon-type cystic degenerative changes. The patient's lower back pain and radiating pain and numbness of both legs improved remarkably postoperatively, and he became asymptomatic at 3 months postoperatively.

Conclusion: Intradural lumbar disc herniation should be highly suspected when intraoperative findings are incompatible with findings from the preoperative imaging examination, and it could be further confirmed via intraoperative ultrasonography and pathological examination of the resected tissue from the dural space. Prompt surgery is recommended, and surgical results are usually favorable. We also reviewed the literature and discussed the potential pathogenesis, natural course, diagnosis, and treatment of intradural lumbar disc herniation.

Keywords: spine, aging population, discectomy, decompression, fixation, fusion

\section{Introduction}

With the growing aging population, more degenerative diseases of the lumbar spine are occurring, among which lumbar disc herniation is the most common. ${ }^{1,2}$ Lumbar disc herniation refers to a disease in which the nucleus pulposus breaks through the weak region of the annulus fibrosus and compresses the nerve roots, causing a series of symptoms. Clinically, most herniated nucleus pulposi are located epidurally, outside or under the posterior longitudinal ligament. Intradural disc herniation (IDDH), when the nucleus pulposus penetrates the posterior longitudinal ligament (PLL) and the anterior wall of the dura and migrates intrathecally, ${ }^{3}$ is very uncommon and accounts for only $0.04-0.33 \%$ of all lumbar disc herniations. ${ }^{4,5}$ The pathogenesis and natural 
course of IDDH remain unclear. In this case study, we describe a patient with lumbar IDDH and review the literature to explore its potential pathogenesis, natural course, diagnosis, and treatment.

\section{Case Description}

A 68-year-old man presented with progressive lower back pain and radiating pain and numbness in both legs for the past 3 years. He did not have symptoms or signs of infectious disease on admission. On physical examination, he showed hypesthesia in the L5 dermatome bilaterally. His muscle strength was normal in both legs. The straight-leg raising sign was positive bilaterally. Radiographs showed a significantly reduced height of the L5-S1 intervertebral space, which was associated with extensive osteophytes localized anteriorly, posteriorly, and laterally on the L5 and S1 vertebral bodies (Figure 1). T2-weighted magnetic resonance imaging (MRI) revealed a large mediolateral herniated disc at L4-L5, resulting in severe spinal stenosis (Figure 2).

After conservative treatment failed, posterior discectomy and fusion of the L4-L5 was scheduled. Intraoperatively, only minimal disc fragments in the epidural space were found after meticulous probing following laminectomy of the L4-L5 vertebrae, and these findings were incompatible with those from the preoperative imaging examination. Additionally, a ruptured PLL and an old tear of the ventral dura were detected. The dorsal dura mater was saturated, tense, and bulged at the L4-L5 levels; moreover, an intradural mass was palpable and confirmed by intraoperative ultrasonography. We thought that the remaining disc fragments could be located in the dura, so we performed dorsal middle durotomy. Upon opening the dural sac, a large cauliflower-like mass similar to nucleus pulposus tissue was found near the arachnoid membrane (Figure 3). The mass was dissociative and could be completely resected. The dorsal dura incision was then closed carefully. Then we removed unilateral articular process joints of L4-L5 to expose and resect the residual L4-L5 disc tissue to avoid L4-L5 disc reherniation, followed by fixation and fusion at the L4-L5 levels to reconstruct the stability of the L4-L5 level (Figure 4). ${ }^{1,2}$ The pathological examination revealed disc tissue with central balloon-type cystic degenerative changes (Figure 5). The patient's lower back pain and radiating pain and numbness of both legs improved remarkably postoperatively, and he was asymptomatic at 3 months postoperatively.
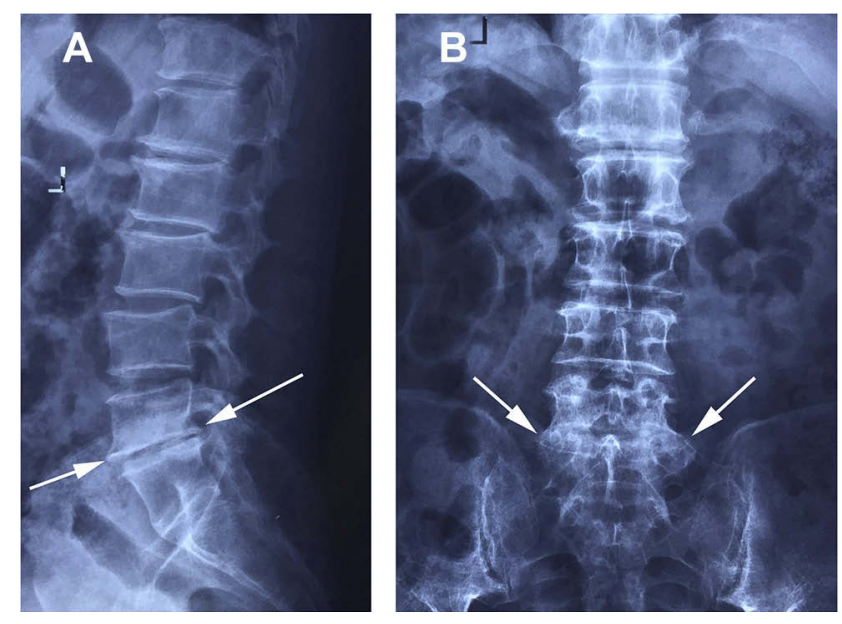

Figure I Radiographs showing advanced lumbar spinal degeneration, with reduced height of the L5-Slintervertebral space (A) and extensive osteophytes (A, B arrows).

\section{Discussion}

As a unique complication of relatively frequent spinal degenerative processes in the elderly population, IDDH has been rarely reported since it was first reported in $1942 .^{6}$ It occurs more commonly in elderly patients, in which the maximum age of patients is 90 years. ${ }^{7}$ Men account for more than $70 \%$ of cases; ${ }^{8} 92 \%$ of cases of IDDH occur in the lumbar spine, $5 \%$ in the thoracic spine, and only $3 \%$ in the cervical spine. ${ }^{9}$

The L4-L5 intervertebral disc region is the most frequently involved segment in lumbar IDDH, ${ }^{10}$ which could be attributed to the following three reasons. First, anatomically, the dura mater of the L4-L5 levels is the closest to the PLL. ${ }^{4}$ Second, L4-L5 intervertebral discs are under the greatest pressure biomechanically. ${ }^{11}$ Third, as the most common segment of lumbar disc herniation, the L4-L5 levels are often associated with annulus fibrosus rupture. ${ }^{12}$ The aforementioned reasons facilitate disc fragments that penetrate the PLL and dural sac. In the present case, IDDH occurred at the L4-L5 levels, which may be related to the above-mentioned reasons.

The pathogenesis and natural course of IDDH is still unclear. ${ }^{13}$ Several hypotheses have been used to explain it. One assumption is the "acute pressure" hypothesis. Sharma et al believed that when the spine suddenly bears external force or when the patient sneezes, the pressure in the intervertebral disc increases sharply. When associated with ruptured annulus fibrosus, the nucleus pulposus may break through the annulus fibrosus, PLL, and dura mater and cause IDDH. Another theory is the "chronic compression" hypothesis. ${ }^{3}$ Lee and Fairholm thought that long-term 

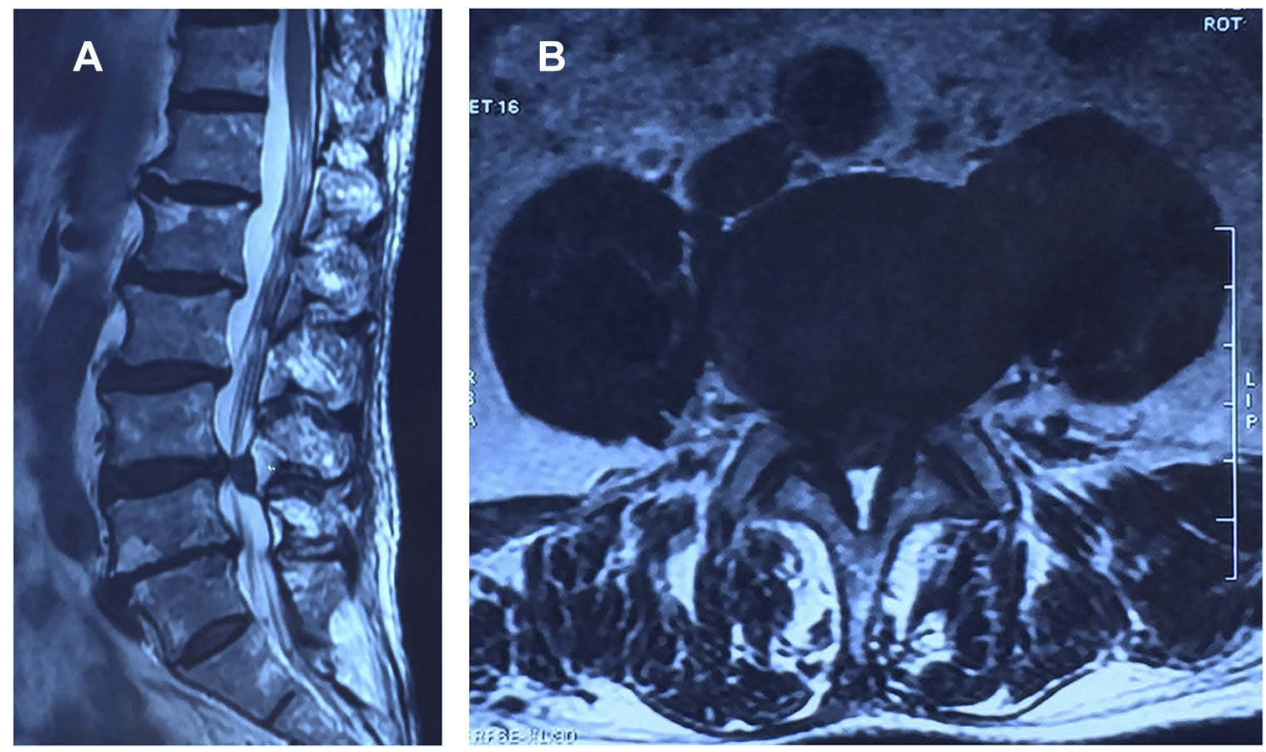

Figure 2 Sagittal (A) and axial (B) magnetic resonance imaging showing a large herniated disc at L4-L5 level.

mechanical compression of structures, such as intervertebral discs and osteophytes, could make the dura mater thinner and fragile, facilitating penetration of the dural sac by the nucleus pulposus. ${ }^{14}$ Besides, the "adhesions" hypothesis is advocated by some researchers. They thought that ventral dural adhesions to the PLL likely play an important role in IDDH. ${ }^{8,15,16}$ The ventral dura and PLL are usually loosely connected, but some risk factors, such as disc herniation, surgical history, and chronic inflammation, can lead to close adhesion among them. Adherent dura becomes thinner under local compression or chronic inflammation and eventually ruptures. In the present case, preoperative disc herniation at the L4-L5 levels, a ruptured PLL, an old ventral tear detected intraoperatively,

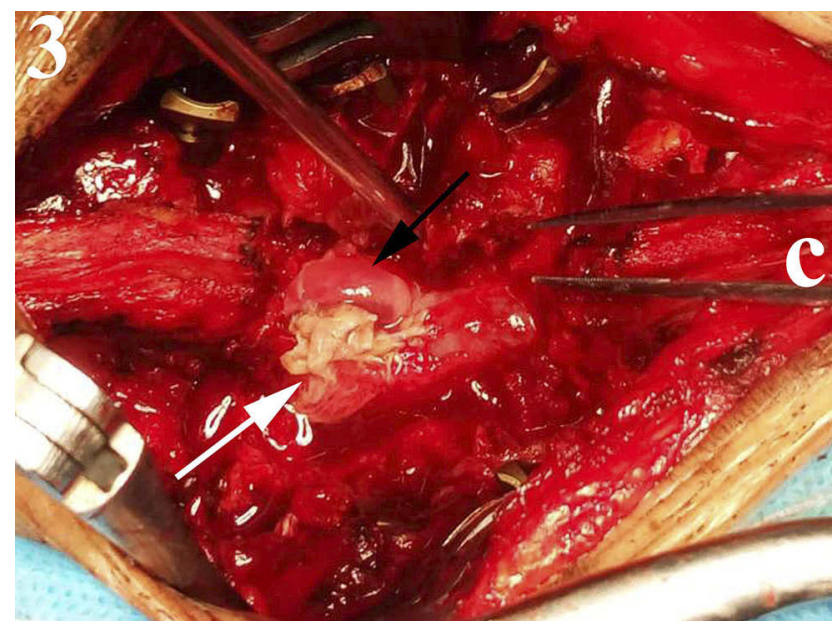

Figure 3 A large cauliflower-like mass (white arrow) was found near the arachnoid membrane (black arrow). $C$ indicates cranial side. and the more than 3 years of pain make us prefer the "chronic compression" and "adhesions" hypotheses rather than the "acute" hypothesis in terms of the pathogenesis and natural course of IDDH.

In terms of the preoperative diagnosis of IDDH, the clinical manifestation of patients and preoperative imaging including radiographs and computed tomography often lack specificity. Patients often present with low back pain and radiating leg pain, similar to lumbar degenerative disease in the elderly population. Preoperative imaging often shows lumbar disc herniation or lumbar spinal stenosis. It is difficult to make an exact diagnosis of IDDH preoperatively by relying on only patient performance and the imaging examination. ${ }^{17,18}$ Wasserstrom et al. reported that IDDH may mimic an intradural tumor; ${ }^{19}$ however, the following MRI findings can distinguish it from the latter: location close to intervertebral disc disease, whorl-like mixed intensity on T2-weighted MRI, poor visualization on T1-weighted MRI, and marked ring enhancement following the administration of gadolinium. ${ }^{12}$ IDDH could enhance MRI because it was surrounded by vascular tissue rich in blood vessels, which was considered to be the most specific imaging finding. ${ }^{19}$ However, IDDH is difficult and seldom suspected preoperatively; thus, there is little opportunity to observe the most specific imaging finding preoperatively, as enhanced MRI is not performed routinely for lumbar degenerative disease.

Probing of the dural sac should be performed intraoperatively if intraoperative findings do not match the preoperative imaging results. The color and toughness of the 


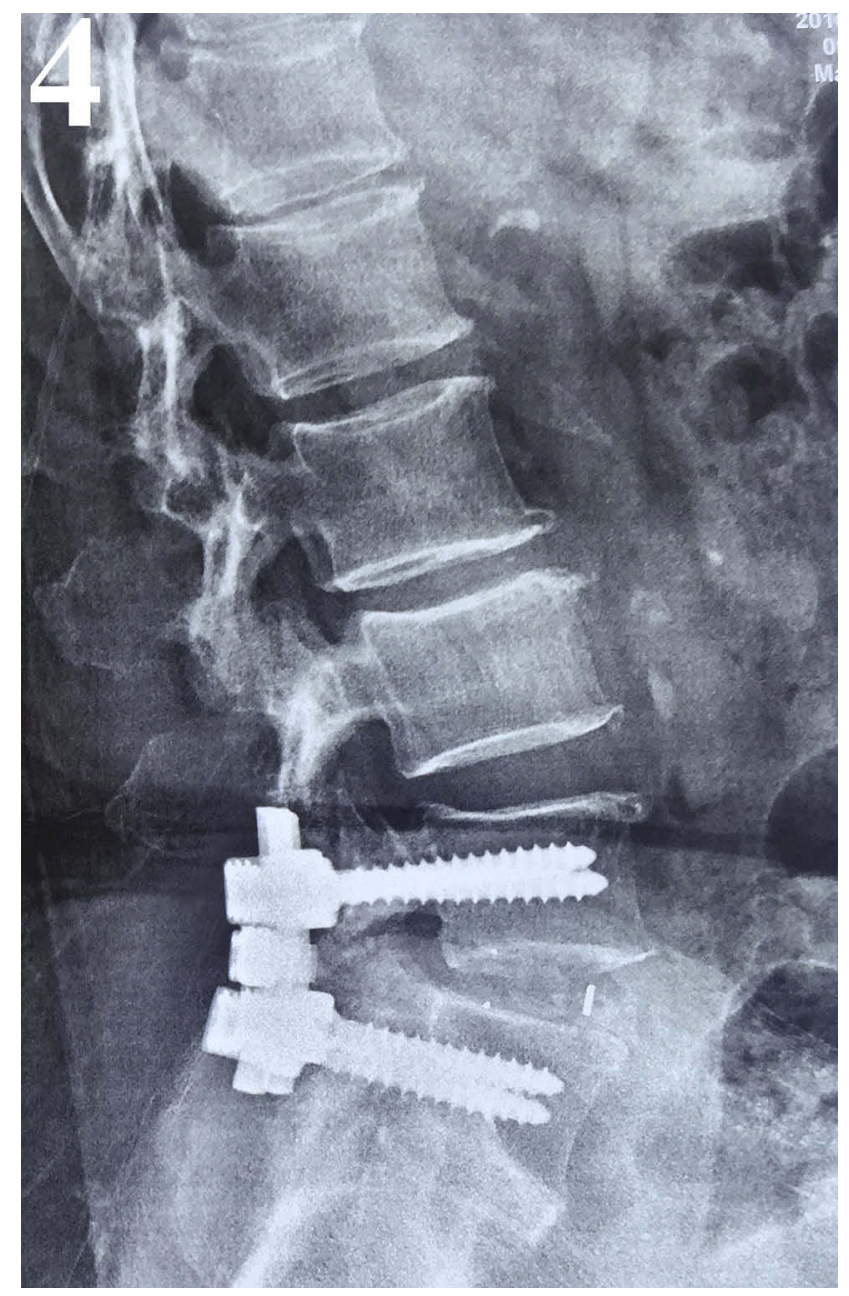

Figure 4 Posterior fixation and fusion at the L4-L5 level.

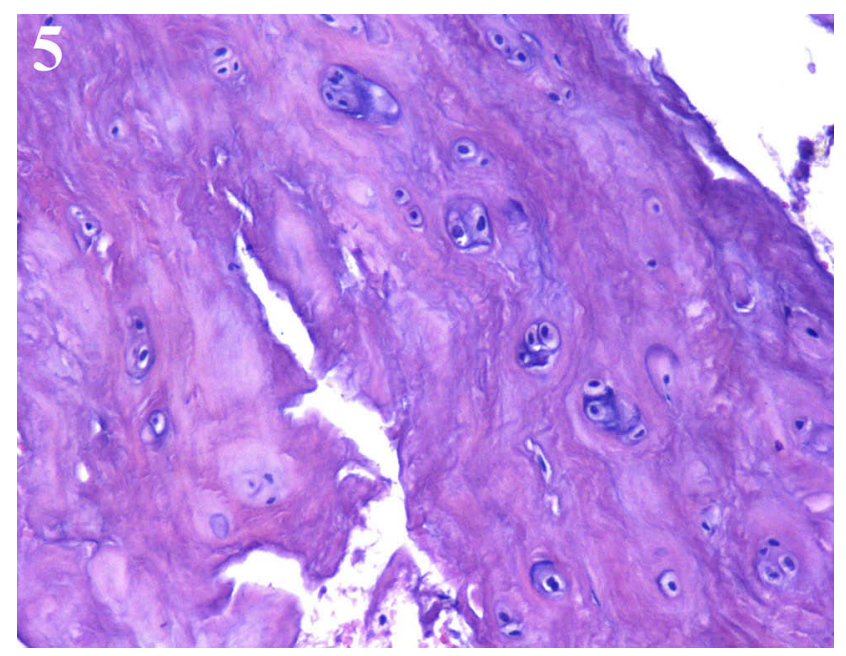

Figure 5 The pathological examination revealing disc tissue (hematoxylin and eosin stain, $\times 100$ )

dura mater should be carefully observed and palpated. Foreign bodies in the dura mater can also be detected by intraoperative ultrasonography. Once IDDH is highly suspected, durotomy must be performed. The pathological examination of the resected tissue in the dural space is the gold standard for diagnosing IDDH.

At present, most surgeons tend to operate as early as possible, and almost all studies emphasize the necessity of early surgery. ${ }^{4,5,8,17} \mathrm{Koc}$ et al considered that prompt surgery was necessary because neurologic prognosis appeared to be closely linked to the preoperative duration of neurologic symptoms. ${ }^{8}$ Montalvo et al advocated early surgical treatment as the standard management of IDDH because of the high incidence of cauda equina syndrome. ${ }^{18}$ Borota et al reported a case of spontaneous resorption of intradural disc fragments. ${ }^{15}$ However, we thought the intradural mass could not be the disc fragments for sure, just depending on MRI in his study. Besides, the patient in his study eventually received the decompression surgery. Thus, despite the significant preoperative neurological deficits, prompt operative treatment of intradural lumbar disc herniation is highly advocated, and its prognosis following surgery is usually favorable.

\section{Conclusions}

It is difficult to make a preoperative diagnosis of IDDH. Therefore, IDDH should be highly suspected and the dural sac should be inspected when intraoperative findings are incompatible with results of the preoperative imaging examination. Prompt decompression surgery is highly recommended, and the surgical results are usually favorable.

\section{Abbreviations}

IDDH, intradural disc herniation; PLL, posterior longitudinal ligament; MRI, magnetic resonance imaging.

\section{Ethics Approval and Informed Consent}

This study was approved by the ethics committee of Xi'an Honghui Hospital, and written informed consent was obtained from the patient to publish the details of his case.

\section{Acknowledgments}

This work was supported by the National Natural Science Foundation of China (grant number 81830077).

\section{Disclosure}

The authors report no conflicts of interest in this work. 


\section{References}

1. Endler P, Ekman P, Berglund I, Moller H, Gerdhem P. Long-term outcome of fusion for degenerative disc disease in the lumbar spine. Bone Joint J. 2019;101-b(12):1526-1533. doi:10.1302/0301620X.101B12.BJJ-2019-0427.R1

2. Chen YC, Zhang L, Li EN, et al. An updated meta-analysis of clinical outcomes comparing minimally invasive with open transforaminal lumbar interbody fusion in patients with degenerative lumbar diseases. Medicine. 2019;98(43):e17420. doi:10.1097/MD.0000 000000017420

3. Sharma A, Singh V, Sangondimath G, Kamble P. Intradural disc a diagnostic dilemma: case series and review of literature. Asian J Neurosurg. 2018;13(4):1033-1036. doi:10.4103/ajns.AJNS_55_17

4. Kataoka O, Nishibayashi Y, Sho T. Intradural lumbar disc herniation. Report of three cases with a review of the literature. Spine. 1989;14 (5):529-533. doi:10.1097/00007632-198905000-00011

5. Schisano G, Franco A, Nina P. Intraradicular and intradural lumbar disc herniation: experiences with nine cases. Surg Neurol. 1995;44 (6):536-543. doi:10.1016/0090-3019(95)00248-0

6. Dandy WE. Recent advances in the diagnosis and treatment of ruptured intervertebral disks. Ann Surg. 1942;115(4):514-520. doi:10.1097/00000658-194204000-00004

7. Orakcioglu B, Dao Trong HP, Jungk C, Unterberg A. Against the odds: massive lumbar intradural disk herniation in the elderly. Global Spine J. 2015;5(5):e84-e87. doi:10.1055/s-0035-1546952

8. Koc RK, Akdemir H, Oktem IS, Menku A. Intradural lumbar disc herniation: report of two cases. Neurosurg Rev. 2001;24(1):44-47. doi:10.1007/PL00011967

9. Epstein NE, Syrquin MS, Epstein JA, Decker RE. Intradural disc herniations in the cervical, thoracic, and lumbar spine: report of three cases and review of the literature. J Spinal Disord. 1990;3 (4):396-403
10. Arnold PM, Wakwaya YT. Intradural disk herniation at L1-L2: report of two cases. J Spinal Cord Med. 2011;34(3):312-314. doi:10.1179/ 2045772311Y.0000000007

11. Yildizhan A, Pasaoglu A, Okten T, Ekinci N, Aycan K, Aral O. Intradural disc herniations pathogenesis, clinical picture, diagnosis and treatment. Acta Neurochir (Wien). 1991;110(3-4):160-165. doi:10.1007/BF01400685

12. Kobayashi K, Imagama S, Matsubara Y, et al. Intradural disc herniation: radiographic findings and surgical results with a literature review. Clin Neurol Neurosurg. 2014;125:47-51. doi:10.1016/j. clineuro.2014.06.033

13. Clatterbuck RE, Belzberg AJ, Ducker TB. Intradural cervical disc herniation and Brown-Sequard's syndrome. Report of three cases and review of the literature. J Neurosurg. 2000;92(2 Suppl):236-240. doi:10.3171/spi.2000.92.2.0236

14. Lee ST, Fairholm D. Intradural rupture of lumbar intervertebral disc. Can J Neurol Sci. 1983;10(3):192-194. doi:10.1017/S03171671 00044905

15. Borota L, Jonasson P, Agolli A. Spontaneous resorption of intradural lumbar disc fragments. Spine J. 2008;8(2):397-403. doi:10.1016/j. spinee.2006.11.004

16. Han IH, Kim KS, Jin BH. Intradural lumbar disc herniations associated with epidural adhesion: report of two cases. $J$ Korean Neurosurg Soc. 2009;46(2):168-171. doi:10.3340/jkns.2009.46.2.168

17. Choi JY, Lee WS, Sung KH. Intradural lumbar disc herniation-is it predictable preoperatively? A report of two cases. Spine J. 2007;7 (1):111-117. doi:10.1016/j.spinee.2006.02.025

18. Montalvo Afonso A, Mateo Sierra O, Gil de Sagredo Del Corral OL, et al. Misdiagnosis of posterior sequestered lumbar disc herniation: report of three cases and review of the literature. Spinal Cord Ser Cases. 2018;4:61. doi:10.1038/s41394-018-0100-9

19. Wasserstrom R, Mamourian AC, Black JF, Lehman RA. Intradural lumbar disk fragment with ring enhancement on MR. AJNR Am J Neuroradiol. 1993;14(2):401-404.
Clinical Interventions in Aging

\section{Publish your work in this journal}

Clinical Interventions in Aging is an international, peer-reviewed journal focusing on evidence-based reports on the value or lack thereof of treatments intended to prevent or delay the onset of maladaptive correlates of aging in human beings. This journal is indexed on PubMed Central, MedLine, CAS, Scopus and the Elsevie
Bibliographic databases. The manuscript management system is completely online and includes a very quick and fair peer-review system, which is all easy to use. Visit http://www.dovepress.com/ testimonials.php to read real quotes from published authors. 